ARRIAGADA, Isabel; FARÍAS, Javiera; WALKER, Agustín: “Evolución de la Población

Penal en Chile desde 1991 a 2007: Aproximación empírica a los efectos de la reforma procesal penal".

Polít. Crim. Vol. 16, № 31 (Junio 2021), Art. 3, pp. 62-82

[http://politcrim.com/wp-content/uploads/2021/04/Vol16N31A3.pdf]

\title{
Evolución de la Población Penal en Chile desde 1991 a 2007: Aproximación empírica a los efectos de la reforma procesal penal*
}

\section{Penal Population Trends in Chile from 1991 to 2007: An Empirical Approach to the Effects of the Chilean Criminal Procedure Reform}

\author{
Isabel Arriagada \\ Abogada, Magíster en Sociología, Doctora (c en Sociología \\ Investigadora Centro de Estudios Justicia y Sociedad. \\ Pontificia Universidad Católica de Chile \\ ilarriagada@uc.cl \\ https://orcid.org/0000-0002-2736-0829
}

\author{
Javiera Farías \\ Abogada, Magíster (C en Criminología y Sociología Jurídico-Penal \\ Universidad de Barcelona \\ jfariape29@alumnes.ub.edu \\ https://orcid.org/0000-0001-6434-2892
}

\author{
Agustín Walker \\ Abogado. Universidad de Chile \\ agustin.walker@derecho.uchile.cl \\ https://orcid.org/0000-0003-2468-6273
}

\section{Resumen}

Fecha de recepción: 18/12/2019.

Fecha de aceptación: 01/09/2020.

La reforma procesal penal (RPP), implementada en Chile a partir del año 2000, constituye la reforma de mayor envergadura en la historia de su sistema de justicia criminal. El presente estudio utiliza un análisis estadístico de regresión lineal para evaluar el impacto de la implementación de la RPP en el período 1991-2007 en las tasas de población penal. Para ello utiliza datos comprehensivos por región, año y régimen procesal, controlando por crecimiento demográfico regional. Los hallazgos indican que ha existido una tendencia sostenida al alza de la población penal condenada que se origina en la década de los noventa, previo a la RPP. Además, la reforma provocó una disminución significativa de la población penal sometida a medidas cautelares privativas de libertad. No existe evidencia para confirmar que la reforma provocó un aumento de la población penal condenada durante el período de estudio. El efecto de la reforma sobre medidas alternativas y el beneficio de libertad condicional es dispar. Estos hallazgos presentan evidencia suficiente para confirmar

\footnotetext{
* Quisiéramos agradecer los valiosos comentarios de Pilar Larroulet, Javier Wilenmann, Diego Rochow, David Jara y Paloma del Villar en la elaboración del artículo. También contamos con la asistencia de Catalina Rufs en la replicabilidad de la construcción de la base de datos.
} 
las tesis existentes sobre la tendencia histórica punitiva en Chile y los efectos garantistas de la reforma procesal penal durante el período de observación.

\begin{abstract}
Criminal justice reform (RPP), implemented in Chile since 2000, constitutes the largest reform in the history of the Chilean criminal procedure. The present study uses a statistical linear regression model to assess the evolution of penal trends and the impact of the implementation of the RPP for the period 1991-2007 through comprehensive data by region, year, and procedural regime, while controlling for regional demographic growth. Findings reveal a sustained upward trend in the convicted prison population that originates in the 1990s, prior to the RPP. In addition, the reform caused a significant decrease in the prison population subjected to pre-trial custody. There is no evidence to suggest that the reform caused an increase in the convicted prison population during the study period. The effects of the reform on alternatives to imprisonment and parole are disparate. These findings provide sufficient evidence to confirm current hypotheses regarding punitive historical trends in Chile and the rights-based effects of the criminal justice reform.
\end{abstract}

Palabras claves: reforma procesal penal, población penitenciaria, encarcelamiento masivo, beneficios penitenciarios, sistema carcelario.

\begin{abstract}
:
Keywords: criminal justice reform, penal population, mass incarceration, alternatives to imprisonment, correctional system.
\end{abstract}

\title{
Introducción
}

El 12 de octubre del año 2000 se publicó la Ley $\mathrm{N}^{\circ}$ 19.696, con la que comenzó en Chile la implementación de la llamada "Reforma Procesal Penal" (en adelante, "la reforma" o "RPP”). La reforma transformó radicalmente el funcionamiento del proceso penal y dejó atrás al obsoleto sistema inquisitivo que imperó en Chile por casi un siglo. El proyecto trajo consigo un nuevo sistema acusatorio y adversarial, la modernización de la administración de los tribunales de justicia y la creación de nuevos servicios públicos, como el Ministerio Público y la Defensoría Penal Pública. ${ }^{1}$ Instauró también nuevos tribunales y procedimientos, consagró nuevas garantías procesales e introdujo modificaciones radicales a instituciones como la prisión preventiva. Su impacto ha sido tan profundo que ha sido calificada como la gran reforma del siglo. ${ }^{2}$

La doctrina en Chile ha discutido extensamente sobre los efectos de la reforma a distintos niveles. Se ha intentado explicar y evaluar sus alcances en distintos ámbitos del quehacer de la justicia criminal; desde su impacto en la cultura judicial ${ }^{3}$ hasta sus efectos en la percepción

\footnotetext{
${ }^{1}$ HORVITZ y LÓPEZ (2005), passim.

${ }^{2}$ LANGER (2007), p. 618.

${ }^{3}$ Por ejemplo, HERSANT (2017), passim.
} 
de inseguridad en la ciudadanía. ${ }^{4}$ En particular, una de las materias respecto de las cuales ha existido intenso debate refiere a las consecuencias de la reforma en las tasas de incremento de la población penal. Frente a ello es posible caracterizar distintas hipótesis. La denominada tesis 'histórica' asevera que ha existido un incremento sostenido de la población penal y una tendencia nacional y global hacia el encarcelamiento masivo desde la década de los 90', cuyo avance no es directamente imputable a la reforma. Por otra parte, los más críticos de la reforma señalan que esta habría provocado un aumento directo de la población penal debido a la mayor agilidad y eficiencia en la operación del sistema con, por ejemplo, la simplificación de procedimientos y reducción de plazos de operación. Esta tesis 'del incremento' sostiene que la reforma ha venido a intensificar el carácter punitivo de las tendencias nacionales anteriores al año 2000. Finalmente, la tesis garantista sostiene que la mayor concesión de garantías al imputado y el establecimiento de medidas cautelares distintas a la prisión preventiva tuvo como consecuencia la disminución de las tasas de población sujeta a medidas cautelares privativas de libertad (población detenida, procesada e imputada). Por lo tanto, independiente de los efectos de la reforma a nivel general, el proyecto entrega una buena noticia a la población sujeta a medidas cautelares. La distinción entre las tres tesis mencionadas no es una contraposición de ideas incompatibles, sino una distinción arquetípica que caracteriza distintos argumentos de la doctrina nacional y sus hipótesis respecto del impacto de la reforma.

El presente estudio levanta información histórica, sistematiza y concuerda las bases de datos contenidas en los Compendios Penitenciarios de Gendarmería de Chile. Para identificar con precisión el efecto de la reforma por región, analizamos tendencias a nivel regional desde los años 1991-2007 y distinguimos según régimen procesal. Para ello, utilizamos datos que abarcan un extenso período de estudio (1991-2007), unidades de análisis (nacional y regional), regímenes (población detenida, procesada, imputada, condenada, con beneficios penitenciarios y libertad condicional) y crecimiento demográfico regional (de acuerdo con los datos del Instituto Nacional de Estadísticas). Para evaluar los efectos de la reforma utilizamos un modelo de regresión lineal que permite testear si la diferencia de la población penal en los períodos pre- y post-reforma es estadísticamente significativa. ${ }^{5}$

Nuestros resultados indican que ha existido una tendencia sostenida al alza de la población penal condenada que se origina en la década de los noventa, previo a la reforma procesal penal. Segundo, los modelos comprueban también que la reforma provocó una disminución significativa de la población penal sometida a medidas cautelares privativas de libertad. En tercer lugar, no existe evidencia suficiente para confirmar que la reforma provocó un aumento de la población penal condenada durante el período de estudio. Finalmente, desde el año siguiente a la implementación de la reforma, se presenta una menor concesión en las medidas de remisión condicional y reclusión nocturna, aunque con un aumento en la aplicación de la libertad vigilada. Estos hallazgos otorgan sustento empírico a la tesis histórica y la tesis garantista, ya que se revela que ha existido un alza de la población penal total desde los 90's,

\footnotetext{
${ }^{4}$ DUCE y PEREZ (2003), passim.

5 Los códigos para este estudio, así como los datos sobre población penal, crecimiento demográfico e implementación de la reforma se encuentran disponibles de manera pública en el repositorio digital: https://github.com/arriagadaisabel/Evolucion-Poblacion-Penal-Chile.
} 
pero que la reforma logró disminuir el número de personas bajo medidas cautelares privativas de libertad. Junto con lo anterior, los hallazgos indican que existen dinámicas dispares respecto de las medidas alternativas al encarcelamiento.

Este estudio representa un avance principalmente metodológico respecto de investigaciones previas. Utilizamos a las regiones como unidad de análisis, distinguimos entre regímenes procesales e identificamos a la población penal detenida, procesada, condenada y sometida a regímenes alternativos. También ampliamos el período de análisis respecto a estudios anteriores (incluyendo datos de inicios de la década de los 90'), controlamos por crecimiento demográfico a nivel regional y utilizamos métodos de comprobación estadística. La sistematización de información ofrecida en el presente artículo alcanza una mayor precisión en la medición de los efectos de la reforma procesal penal, el análisis de las fluctuaciones de la población penal en Chile y permite verificar empíricamente las teorías en la materia. El estudio, sin embargo, tiene varias limitaciones que detallamos más adelante. Futuras investigaciones tienen amplio espacio para estudiar con mayor precisión los efectos de la reforma.

\section{El impacto de la reforma procesal penal en Chile: Tres tesis divergentes}

\subsection{La tesis histórica}

En su texto, “¿Por qué aumenta la población penal en Chile? Un estudio criminológico longitudinal”, el investigador Sebastián Salinero analiza las causas del incremento de la población carcelaria en Chile durante el período 2000-2009. El estudio, que enfatiza especialmente los efectos de la reforma procesal penal, ha servido de base para múltiples análisis sobre la evolución de la población penal chilena y la reflexión sobre las condiciones estructurales, legislativas y políticas que han promovido esta expansión. El autor asevera que el incremento de la población penitenciaria en Chile es una tendencia que se inicia a lo menos desde la década de los $80^{\prime} .{ }^{6}$ Esta explicación coincide con lo planteado por buena parte de la literatura nacional, la cual asevera que el crecimiento del número de condenados en Chile se remonta a los orígenes de la dictadura (1973), y a los inicios del período de transición a la democracia (1990), fruto del auge del discurso de la seguridad ciudadana ${ }^{7}$ y la politización del problema de la delincuencia. ${ }^{8}$ Los mecanismos concretos de expansión penal apuntan a la tipificación de nuevas conductas delictivas, aumento y extensión de penas existentes ${ }^{9}$, la restricción en el acceso a alternativas a las penas privativas de libertad ${ }^{10}$ y libertades condicionales. ${ }^{11}$ Bajo esta perspectiva, nuevas transformaciones institucionales, como la reforma procesal penal, vienen a intensificar el flujo de ingreso de personas al sistema penitenciario. Sin suficientes mecanismos de descompresión, ${ }^{12}$ el sistema penitenciario

${ }^{6}$ SALINERO (2012), p. 116.

${ }^{7}$ DAMMERT (2005), p. 24.

${ }^{8}$ CUNEO (2018), p. 184.

${ }^{9}$ MORALES (2012), p. 104.

${ }^{10}$ SALINERO (2012), p. 141.

${ }^{11}$ En Chile, véanse las leyes $\mathrm{N}^{\circ} 19.413$ de 1995, No 19.366 de 1995, № 19.449 de 1996, № 19.617, de 1999, entre otras. Sobre otras posibles causas de su disminución, SALINERO (2012), pp. 142 y ss.

${ }^{12}$ SALINERO (2012), pp. 140 y ss. 
chileno habría desarrollado un esquema estructural e histórico hacia la expansión. En este contexto, la reforma procesal penal habría contribuido a intensificar una tendencia de larga data.

Esta tendencia descrita por la tesis histórica no es exclusiva del contexto chileno. Ella pertenecería al fenómeno global de encarcelamiento masivo y del uso de la herramienta penal como respuesta privilegiada al fenómeno del delito. ${ }^{13}$ Entre las posibles causas de estas tendencias, se apunta al auge y consolidación global del denominado populismo punitivo ${ }^{14}$ como mecanismo de gestión de la inseguridad ciudadana y el temor colectivo. La necesidad de las autoridades políticas de reforzar su legitimidad soberana a través de mensajes punitivos habría conllevado a un aumento sustantivo del uso de la herramienta penal desde los años ochenta. ${ }^{15}$

\subsection{La tesis 'del incremento'}

La reforma procesal penal fue fruto de un consenso entre sectores que buscaban aumentar la eficacia del sistema penal y grupos progresistas que aspiraban a modernizar el proceso penal y consagrar mayores garantías para los imputados y condenados de acuerdo con estándares constitucionales e internacionales vigentes. ${ }^{16} \mathrm{La}$ tesis 'del incremento' atiende al primero de estos objetivos, pues sostiene que la implementación de la reforma procesal penal en Chile produjo un aumento especial en la población penal debido a la introducción de mecanismos que optimizaron la eficacia del sistema en la persecución del delito.

Silvio Cuneo, en su artículo "Encarcelamiento en Chile. Necesidad de una nueva regulación a nivel constitucional", sitúa a la reforma procesal penal como una de las causas inmediatas del encarcelamiento masivo en Chile. ${ }^{17}$ Ello, explica Cuneo, debido a los incentivos de admisión de culpa de los procedimientos simplificados y abreviados y la expansión de poderes del Ministerio Público y las policías. ${ }^{18}$ En una línea similar de análisis, el académico en estudios socio legales, Javier Wilenmann, entrega evidencia agregada sobre tasas de encarcelamiento a nivel nacional y sugiere que la reforma procesal penal gatilló la optimización de la imposición de sanciones penales y, por tanto, contribuyó a un aumento de la población penal. ${ }^{19}$ Los postulados de ambos autores presentan, sin embargo, diferencias

\footnotetext{
${ }^{13}$ RIVERA (2017), pp. 47 y ss. En el mismo sentido, GARLAND (2001), p. 1.

${ }^{14}$ LARRAURI (2006), p. 129.

15 TORRES (2015), p. 17; LARRAURI (2006), p. 18. En el mismo sentido, DAMMERT (2005), p. 41. ZIMRING (2001), p. 145. También, GARLAND (2001), passim. Dicha tendencia también se da, con menor intensidad, en Europa. Así: GARCÍA (2019), p. 99; LARRAURI (2009), p. 4; y DOWNES (2001), p. 57.

${ }^{16}$ MORALES (2012), p. 103.

${ }^{17}$ Salinero y Cuneo coinciden en gran parte de su análisis. La diferencia en las tesis de cada uno se refiere únicamente al período de análisis y, en relación con ello, a la incidencia que le otorgan a la implementación de la Reforma Procesal Penal. Ambos coinciden en que el aumento de la población penal es un fenómeno multicausal que se relaciona con factores político-sociales y con la dictación de leyes penales que han endurecido las sanciones. Coinciden también en descartar que este aumento de deba a un incremento de delitos. Con respecto a los efectos de la Reforma Procesal Penal, ambos afirman que esta constituye una causa del aumento de la población penal, sin embargo, Salinero extiende el período de análisis, lo que le permite concluir que la tendencia creciente es previa y que se sitúa a inicios de los años 80 '.

${ }^{18}$ CUNEO (2008), pp. 1-10.

${ }^{19}$ WILENMANN (2019), p. 7.; AREVALO (2018), p. 50.
} 
con relación a los factores que explican este aumento y, en particular, al contexto en el cual se enmarca la reforma. Para Cuneo, las causas mediatas, a nivel global, de este encarcelamiento masivo — que en Chile se concreta mediante la reforma y la dictación de legislación sustantiva que endurece las sanciones penales - se hallan en el desmantelamiento del Estado Social y del ideal rehabilitador, junto con el advenimiento de políticas públicas inspiradas en el populismo punitivo. Wilenmann, en cambio, advierte que la perspectiva de análisis basada en la sustitución del estado de bienestar por modelos económicos de corte neoliberal (la tesis neoliberal en palabras de este autor), no es directamente aplicable a la región ni tiene suficiente poder explicativo para dar cuenta de los cambios experimentados en el sistema penal, puesto que ni en Chile ni en el resto de los países de Latinoamérica se existió una estructural estatal de Estado de Bienestar. Así, Wilenmann atribuye la expansión penal al mejoramiento de la eficacia en la provisión de control social. Esta expansión perpetúa, antes que sustituye, la tendencia histórica de control social de la población marginada mediante su exclusión física y confinamiento.

La tesis 'histórica' y 'del incremento' son perfectamente compatibles. La tesis del incremento no niega la posibilidad de que la población penal hubiese experimentado un aumento sostenido previo a la reforma. Sin embargo, afirma también que la reforma vino a contribuir a una expansión de la población penal de mayor intensidad, la que presentaría tasas de crecimiento superiores a las de períodos anteriores.

\subsection{La tesis garantista}

La tesis garantista enfatiza los beneficios de la reforma procesal penal respecto de la población detenida y sometida a prisión preventiva. Esta postura atiende a que uno de los principales problemas del sistema inquisitivo previo a la reforma apuntaba a la desproporción que existía entre una extensa población penal procesada no condenada y la población con condena efectiva. ${ }^{20}$ Dentro del marco del antiguo Código de Procedimiento Penal, el denominado 'auto de procesamiento' derivaba automáticamente en la privación de libertad del procesado como medida de custodia. Ante esta circunstancia, el mensaje ejecutivo del Presidente Eduardo Frei en la presentación del proyecto de reforma ya en 1995 aseveraba: "la mayor parte de quienes están recluidos en las prisiones del país son personas que se encuentran detenidas o procesadas y sólo la menor parte condenadas." ${ }^{21}$ La preocupación de las autoridades de la época atiende a la necesidad de racionalizar el uso de las medidas cautelares personales y respeto a los principios de excepcionalidad y presunción de inocencia. ${ }^{22} \mathrm{El}$ programa de la reforma involucraba entonces imponer mayores restricciones a la judicatura para someter a los infractores de ley a una medida cautelar privativa de libertad. Esto en consonancia también con los estándares internacionales que crecientemente empezaban a dominar el concierto latinoamericano durante la transición a la democracia. ${ }^{23}$

\footnotetext{
${ }^{20}$ BAYTELMAN y DUCE (2003), passim.

${ }^{21}$ Historia de la Ley 19.696 , p. 5

22 VENEGAS y VIAL (2008), passim.

23 TIEDE (2012), p. 86.
} 


\section{Polít. Crim. Vol. 16, № 31 (Junio 2021), Art. 3, pp. 62-82 [http://politcrim.com/wp-content/uploads/2021/04/Vol16N31A3.pdf]}

Bajo esta hipótesis "garantista" la reforma procesal penal habría conllevado a la concesión de mayores derechos a la población sometida a control penal previo a la condena, lo que a su vez puede haber derivado en una disminución de la población detenida, procesada e imputada. Los mecanismos de disminución en este caso apuntan a que la reforma involucró el establecimiento de medidas cautelares personales distintas a la prisión preventiva (artículo 155 del Código procesal penal), un mayor respeto al principio de presunción de inocencia, mayor control del ente persecutor sobre las facultades policiales, mayores restricciones sobre el denominado 'auto de procesamiento' y el establecimiento de una formación garantista de los operadores del sistema. Lydia Brashear Tiede en su estudio cuantitativo sobre los efectos de la Reforma Procesal Penal utiliza un modelo estadístico longitudinal y demuestra que la reforma efectivamente provocó una disminución significativa de la población penal chilena no condenada y privada de libertad, específicamente, procesados y detenidos. La evidencia empírica existente, por tanto, también contribuye a respaldar esta hipótesis. ${ }^{24}$

\subsection{Síntesis}

La tesis histórica caracteriza al sistema penal chileno por su tendencia crónica al punitivismo desde, al menos, la década de los 80'. La tesis 'del incremento', por otra parte, enfatiza el rol de la reforma procesal penal como factor decisivo de la radical expansión de la población penal condenada en Chile. La tesis garantista argumenta que al menos la población detenida y procesada se vio beneficiada con la reforma procesal penal. Así entonces, existen tres tesis arquetípicas respecto de los efectos de la reforma procesal penal: la tesis histórica, la tesis del incremento y la tesis garantista.

Las tesis expuestas no son necesariamente contradictorias. Más bien, es posible afirmar que iluminan distintos aspectos en los cuales incidió la implementación de la reforma, sea en el cumplimiento de los objetivos para los que fue creada o las tendencias preexistentes que contribuyó a profundizar. Así, bien puede acontecer que (i) al aumento progresivo de la población penal condenada acontezca desde los años ochenta, (ii) a ello se le acompañe una expansión de la población penal de mayor intensidad luego de la reforma (iii) lo anterior, en conjunto con la disminución de la población penal procesada y detenida (no condenada). Ciertamente, la disminución de la población procesada puede o no ser concomitante al aumento de la población condenada. También puede suceder que la población procesada sea 'gestionada' por el sistema con mayor celeridad y, por lo tanto, aumente significativamente la población condenada debido a esta mayor capacidad del sistema para condenar. En suma, las tesis pueden coincidir y conformar, en conjunto, el panorama global de análisis de los efectos de la reforma.

Hasta ahora existe escasa evidencia que permita demostrar estadísticamente la plausibilidad de estas tres posturas. La mayoría de los estudios existentes utiliza datos agregados a nivel nacional y por un período que comprende solo los años 2000 y siguientes. $^{25}$ Esta circunstancia impide evaluar con precisión la naturaleza escalonada de la implementación de la reforma a nivel regional, así como tendencias de larga data previas a la reforma.

\footnotetext{
${ }^{24}$ En este mismo sentido: DUCE y RIEGO (2016), p. 174.

${ }^{25}$ Por ejemplo: Salinero (2012), passim. Una afortunada excepción es WILENMANN (2019), passim.
} 
Utilizar solo cifras a nivel nacional para medir el efecto de la reforma procesal penal es un ejercicio con serias limitaciones. En primer lugar, cada región tiene sus propias características e historia, por lo tanto, los análisis con cifras nacionales resultan insensibles a las dinámicas regionales particulares. En segundo lugar, en datos agregados a nivel nacional, los promedios varían enormemente dependiendo de la forma en que se manejen los datos y de las bases de datos que se utilice. ${ }^{26}$ Por ejemplo, diversos estudios utilizan el reporte del World Prison Brief preparado por el Institute for Crime \& Justice Policy Research para analizar tendencias de población penal. El problema es que su construcción del promedio nacional trata como una sola cifra a los internos en prisión preventiva e internos sujetos a medidas cautelares privativas de libertad distintas de la prisión preventiva. ${ }^{27}$ En tercer lugar, para el caso puntual de la reforma procesal penal, debemos considerar que esta tuvo una implementación escalonada en el territorio nacional desde el año 2001 en las regiones de Coquimbo y de la Araucanía hasta el año 2005 en la Región Metropolitana. Por lo tanto, el período de impacto de la reforma difiere por región (Tabla 1).

Tabla 1. Calendario de implementación de la reforma procesal penal

\begin{tabular}{llcc}
\hline Fecha de implementación & Región & Período de adaptación & Período de consolidación \\
\hline Diciembre, 2000 & 4 y 9 & $2001-2002$ & 2003 y siguientes \\
Octubre, 2001 & 2,3 y 7 & $2002-2003$ & 2004 y siguientes \\
Diciembre, 2002 & 1,11 y 12 & $2003-2004$ & 2005 y siguientes \\
Diciembre, 2003 & $5,6,8$ y 10 & $2004-2005$ & 2006 y siguientes \\
Junio, 2005 & Región Metropolitana & $2006-2007$ & 2008 y siguientes \\
\hline Tabla 1: Calendario de Reforma. Fuente Tiede, Lydia Brashear. "Chile's Criminal Law Reform: Enhancing Defendants' \\
\multicolumn{4}{r}{ Rights and Citizen Security." Latin American Politics and Society 54, no. 3 (2012): 65-93. }
\end{tabular}

Para sortear esta dificultad, nuestro estudio evalúa el efecto de la reforma en cada una de las regiones del territorio nacional por medio de un modelo estadístico de regresión que compara las diferencias en la población penal pre- y post reforma considerando el año de implementación por región. Gracias a que nuestros datos comprenden los años 1990 a $2007^{28}$, logramos identificar las tendencias de la evolución de la población penal durante todo el período de transición a la democracia y capturar el efecto de la reforma hasta el año de adaptación de la última región en que fue implementada (Región Metropolitana). Así, nuestros datos permiten evaluar la plausibilidad de las tesis 'garantista', 'del incremento' e 'histórica' con mayor precisión que estudios anteriores y distinguir el efecto de la implementación de la reforma per se. Nuestros datos no permiten capturar el efecto de la expansión de la reforma, modificaciones legislativas posteriores ni el proceso de aprendizaje institucional posteriores al año 2007. ${ }^{29}$

En lo que sigue, el artículo se organiza en cuatro secciones. En la primera sección describimos los datos disponibles y las dificultades de la sistematización de la información

\footnotetext{
${ }^{26}$ HOLDERNESS (2016), passim.

${ }^{27}$ Una afortunada excepción es WILENMANN (2019), passim.

${ }^{28}$ Como explicaremos más adelante, esta restricción se debe a las modificaciones administrativas motivadas por la creación de las regiones de Arica y Ñuble.

${ }^{29}$ Agradecemos a Javier Wilenmann y a los pares evaluadores por hacernos notar estos puntos.
} 
contenida en los Compendios Estadísticos Penitenciarios del Servicio de Gendarmería de Chile. La segunda sección describe la metodología de análisis. La tercera sección resume las principales tendencias nacionales y regionales y analiza la plausibilidad de las tesis existentes a través de un modelo estadístico. La sección final del artículo resume las conclusiones, limitaciones y recomendaciones de nuestro estudio.

\section{Información disponible: Los Compendios Estadísticos Penitenciarios}

La información estadística sobre evolución de la población penal en Chile se encuentra dispersa y fragmentada en diversas fuentes. Estudios existentes han utilizado información proveniente, por una parte, de servicios públicos como el Ministerio Público, el Instituto Nacional de Estadísticas y el Ministerio del Interior, y por otra, de organizaciones privadas, como Fundación Paz Ciudadana. ${ }^{30}$ Nuestro estudio utiliza exclusivamente información proveniente del Servicio de Gendarmería de Chile y sus Compendios Estadísticos Penitenciarios (en adelante, los "compendios penitenciarios" o "compendios"). Estos compendios demuestran el esfuerzo del servicio de Gendarmería de Chile por sistematizar la información sobre la población penal. Gendarmería de Chile es el ente custodio de la población penal, contabiliza diariamente la población sometida a su tutela y es, por tanto, la única institución que mantiene un completo registro de cada una de las personas privadas de libertad en Chile. Sus reportes son utilizados por organizaciones internacionales, como el referido World Prison Brief, para retratar las tendencias en la población penal chilena. Mientras instituciones como el poder judicial contabilizan causas o sentencias, Gendarmería de Chile contabiliza 'personas'. Por lo mismo, otros registros suelen ser imprecisos, puesto que su objeto de referencia no captura necesariamente el flujo diario de la población privada de libertad. Los compendios constituyen una valiosa fuente de información y probablemente representen el recurso más confiable sobre la evolución de la población penal chilena.

Con todo, los datos de Gendarmería para el período bajo estudio distan de ser perfectos. En el proceso de sistematización, identificamos tres problemas principales. Primero, los compendios utilizan términos y referencias de manera imprecisa, lo que dificulta la comprensión de la base de datos e impide capturar la variación en las tendencias en distintos regímenes procesales. Por ejemplo, algunos compendios registran cifras correspondientes al último día del período analizado, mientras que en otros se contabilizan los promedios anuales. A ello se suma que los registros presentan información inconsistente o contradictoria. Así, por ejemplo, en ciertos años la suma de totales regionales no coincide con los totales señalados a nivel nacional. Otro problema frecuente es la utilización de distintos indicadores para medir un mismo concepto. Por ejemplo, desde el año 2001 individuos condenados a arresto nocturno empiezan a contabilizarse dentro de la población recluida, a diferencia de años anteriores, en que se les consideraba como sujetos a beneficios de reinserción, lo que altera la contabilización del total de reclusos y, en particular, la de condenados. Finalmente, ciertas sumatorias que permanecen consistentes durante ciertos períodos para el cálculo de la población penal total pierden consistencia en años subsiguientes.

\footnotetext{
${ }^{30}$ Por todos: SALINERO (2012), passim.
} 
Para sortear las inconsistencias utilizamos dos estrategias. En primer lugar, acudimos al desglose de datos generales contenidos en los anexos de los compendios. Quien haya accedido a los compendios puede observar que el cuerpo del documento presenta información agregada, habitualmente clasificada por régimen procesal. En cambio, nuestra base de datos acude a los datos desglosados y desagregados según región y régimen procesal. En segundo lugar, cruzamos sumatorias nacionales y regionales de modo tal que la suma de todas las cifras regionales coincidiera con la sumatoria que registra el compendio a nivel nacional. La Tabla 2 enumera las inconsistencias encontradas en el compendio y la cifra de reemplazo, que denominamos cifra concordada. Así, nuestro estudio concuerda y sistematiza esta información, utiliza consistentemente la contabilización de promedios anuales, y usa definiciones estables para identificar los regímenes procesales en estudio.

Tabla 2. Inconsistencias encontradas en compendios penitenciarios elaborados por

\begin{tabular}{ccccc}
\multicolumn{5}{c}{ Gendarmería de Chile. } \\
\hline Año & \multicolumn{4}{c}{$\begin{array}{c}\text { Cifra original en el } \\
\text { compendio }\end{array}$} \\
\hline 1991 & Régimen & Región & $\begin{array}{c}\text { Cifra } \\
\text { concordada }\end{array}$ \\
1991 & Detenidos & 8 & 388 & 382 \\
1991 & Lib. Condicional & 5 & 411 & 410 \\
1991 & Lib. Condicional & 6 & 121 & 111 \\
2000 & Prondicional & 8 & 629 & 630 \\
2000 & Procesados & 8 & 1537 & 1540 \\
2000 & Remisión & 9 & 464 & 463 \\
& & 12 & 428 & 498 \\
\hline
\end{tabular}

En segundo lugar, la información existente para el período 1991-2007 no se encuentra digitalizada, lo que dificulta la manipulación de datos y la elaboración de gráficos. Nuestro trabajo comprendió la digitalización de la información disponible y la creación de una matriz de datos.

Finalmente, el cuerpo de los compendios penitenciarios sistematiza información a nivel regional. El problema de esta aproximación se debe a que la reorganización administrativa del año 2008 y creación de la región de Arica y Parinacota y la región de Los Ríos impide comparar tendencias regionales post- 2008. Por esta razón, nuestro estudio se restringe al periodo 1991-2007 y utiliza las - en ese entonces - trece regiones chilenas como unidad de análisis. Nuestra sistematización organiza e imputa los datos de manera de proveer coherencia y sentido a la información disponible. Finalmente, como es práctica habitual en el estudio de población penal ${ }^{31}$, utilizamos datos del Instituto Nacional de Estadísticas para distinguir el crecimiento de la población penal con el crecimiento demográfico regional y construir tasas comparables. ${ }^{32}$

\footnotetext{
${ }^{31}$ Ver, por ejemplo, WILENMANN (2019), passim.

${ }^{32}$ Por eso utilizamos la fórmula de las tasas consistente en $\mathrm{t}=($ población penal/ población regional)*100.000.
} 


\section{Metodología}

Nuestro análisis primero atiende a la descripción visual de las tendencias nacionales y regionales. Luego, para testear estadísticamente la información disponible, utilizamos un modelo estadístico de regresión lineal basado en una estructura de datos de panel. Gracias a esta aproximación, nuestro modelo permite comparar las diferencias de la población penal en distintos regímenes en los períodos pre- y post-reforma en regiones sin reforma y con reforma. Para ello utilizamos los comandos xtset y xtreg del software Stata 16 ().

\section{Resultados y análisis}

\subsection{Tendencias nacionales}

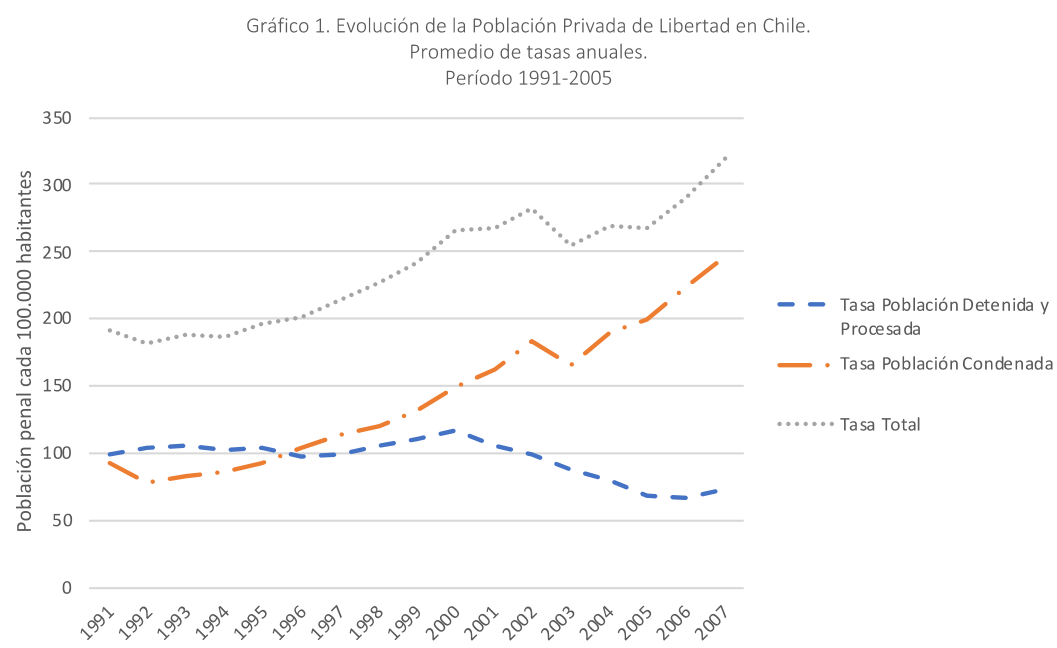

Fuente: Elaboración propia sobre la base de los Compendios Penitenciarios de Gendarmería de Chile

El Gráfico 1 exhibe las tendencias nacionales anuales de la población penal recluida, con detalle en la población sujeta a medidas cautelares privativas de libertad y condenada a pena privativa de libertad durante los años 1991 a 2007. Para efectos del análisis, entenderemos como población sometida a medidas cautelares privativas de libertad a la población detenida, procesada e imputada. La denominación 'imputado' emerge con la reforma procesal penal.

Es posible observar que, durante el período 1991 a 1995, la tasa de población sujeta a medidas cautelares privativas de libertad es mayor a la tasa de población condenada con pena privativa de libertad. La tasa de población cautelar muestra una tendencia estable de entre 90 a 120 detenidos y procesados hasta el año 2000 , momento en el cual empieza a exhibir un sostenido declive. La tasa de población condenada aumenta casi ininterrumpidamente desde los años 1991 a 2007 desde 70 hasta más de 200 condenados por cada 100.000 habitantes; solo con una breve caída durante el período 2002 y 2003. La tasa total de población privada de libertad (suma de medida cautelar y condenada) muestra un aumento creciente y casi ininterrumpido en el período de observación. Nuestros datos coinciden con estudios previos. 

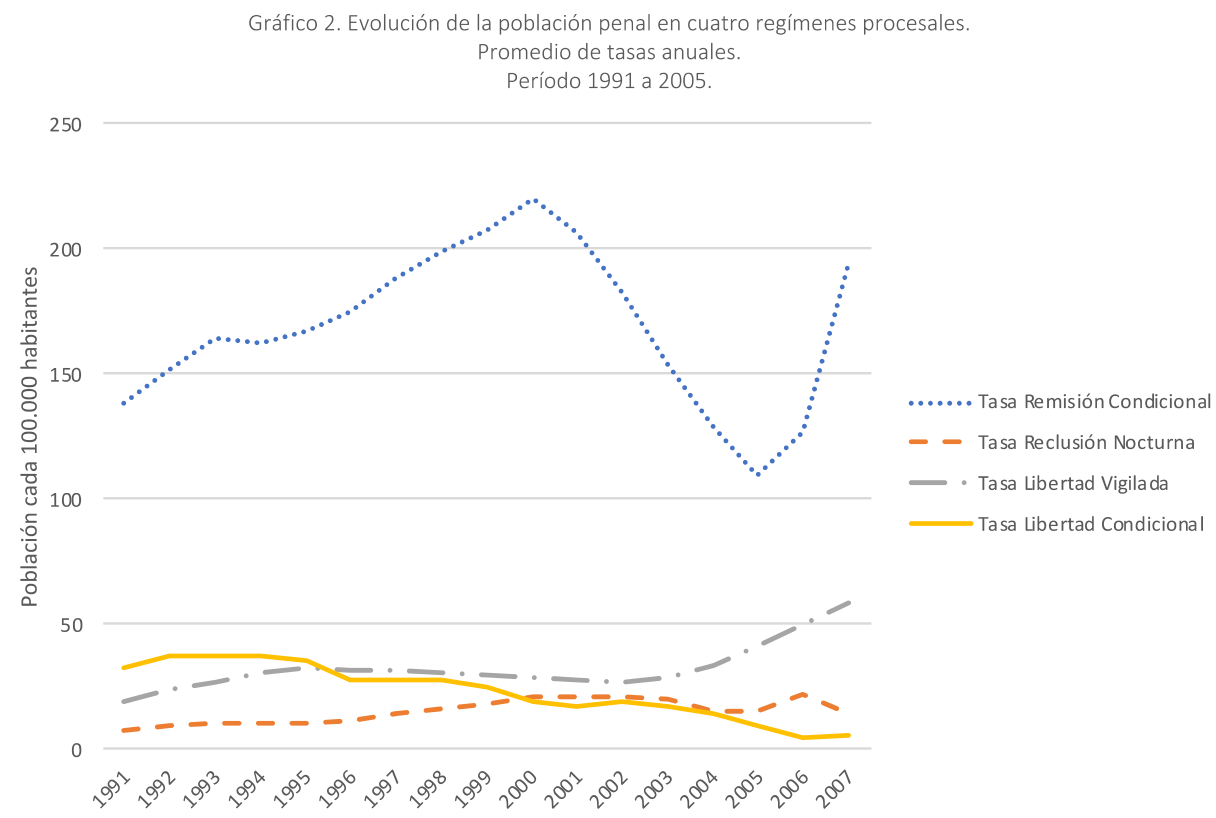

Fuente: Elaboración propia sobre la base de los Compendios Penitenciarios de Gendarmería de Chile

El Gráfico 2 exhibe las tendencias nacionales anuales de la población penal no sujeta a privación de libertad total. Distinguimos entre población sujeta a remisión condicional, reclusión nocturna, libertad vigilada y libertad condicional. La población sujeta a reclusión nocturna se mantiene constante en menos de 20 reclusos por cada 100 mil habitantes. La población bajo libertad vigilada aumenta sostenidamente desde alrededor de 20 a más de 50 personas por cada 100 mil habitantes. La mayor variación se presenta en el régimen de remisión condicional de la pena, que puede dividirse en tres etapas. Primero, hay una tendencia de alza desde 1990 hasta el año 2000, donde la población bajo remisión condicional alcanza un máximo de casi 220 remitidos por cada 100 mil habitantes. Desde el año 2000 en adelante, la medida presenta un franco declive y alcanza su nivel más bajo el año 2005. Luego experimenta un aumento sostenido durante el período 2005 a 2007.

Esta primera aproximación gráfica provee un indicio hacia las tendencias de evolución de la población penal en Chile. Sin embargo, reiteramos que esta aproximación no captura las posibles diferencias contextuales de cada región. Esto es particularmente relevante, pues una aproximación a la variación local es crucial si se considera que la reforma procesal penal tuvo una implementación escalonada y que cada región tiene sus propias dinámicas en materia penitenciaria. Por esta razón, la siguiente sección resume tres marcadas tendencias que identificamos a nivel regional. 


\subsection{Tendencias regionales}

Gráfico 3. Evolución de la población privada de libertad en 13 regiones

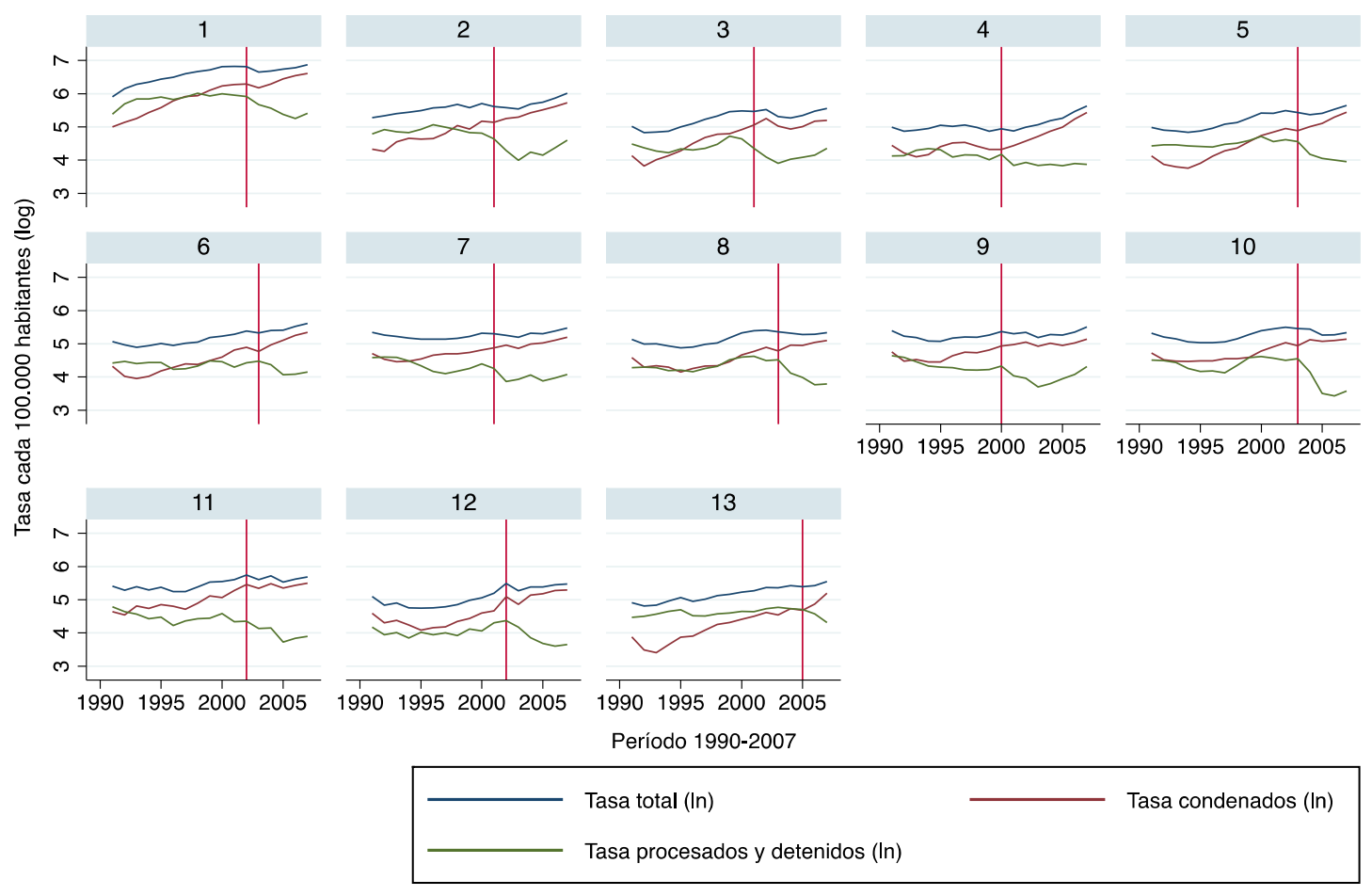

Línea de referencia: Año de implementación de la reforma procesal penal

Fuente: Elaboración propia sobre la base de los Compendios Penitenciarios de Gendarmería de Chile e Instituto Nacional de Estadísticas

El gráfico 3 ilustra la evolución de las tasas de población penal sometida a medidas cautelares privativas de libertad (detenidos, procesados e imputados), las tasas de población penal condenada privada totalmente de libertad (condenados) y la suma de ambas tasas (población penal total). Para hacer comparables las mediciones entre regiones debimos hacer una conversión al logaritmo de la tasa, como es práctica estándar en este tipo de estudios. ${ }^{33}$ En cuanto a las tasas de población penal condenada, todas las regiones exhiben una tendencia al alza que se origina en el inicio de la década de los noventa y se mantiene más o menos consistente hacia el final del periodo de observación. Este hallazgo sugiere que el aumento de la población condenada tiene lugar desde el inicio de la década de los noventa y provee evidencia preliminar a favor de la tesis histórica. En cuanto a las tasas de población detenida, procesada e imputada (analizada conjuntamente como población 'cautelada'), las imágenes parecen indicar que todas las regiones experimentan un descenso hacia el final del período de observación. Un dato importante es que, al inicio de la ventana de observación, la población penal cautelada es mayor a la población penal condenada en la mayoría de las regiones $(1,2,3,5,6,10$ y 13) y con mayor intensidad en la Región Metropolitana (13). Al final del período, todas las regiones muestran una población condenada superior a la población sujeta a medidas cautelares privativas de libertad. Por lo demás y en consistencia

${ }^{33}$ Por todos TIEDE (2012), passim. 
con la literatura, los datos muestran que la primera región presenta la mayor tasa de población penal durante todos los años en observación. ${ }^{34}$

En resumen, el Gráfico 3 entrega importantes indicios de la tendencia regional generalizada al aumento sostenido del encarcelamiento durante la década de los noventa ${ }^{35}$. Sin embargo, no proporciona evidencia suficiente para confirmar el impacto de la reforma procesal penal.

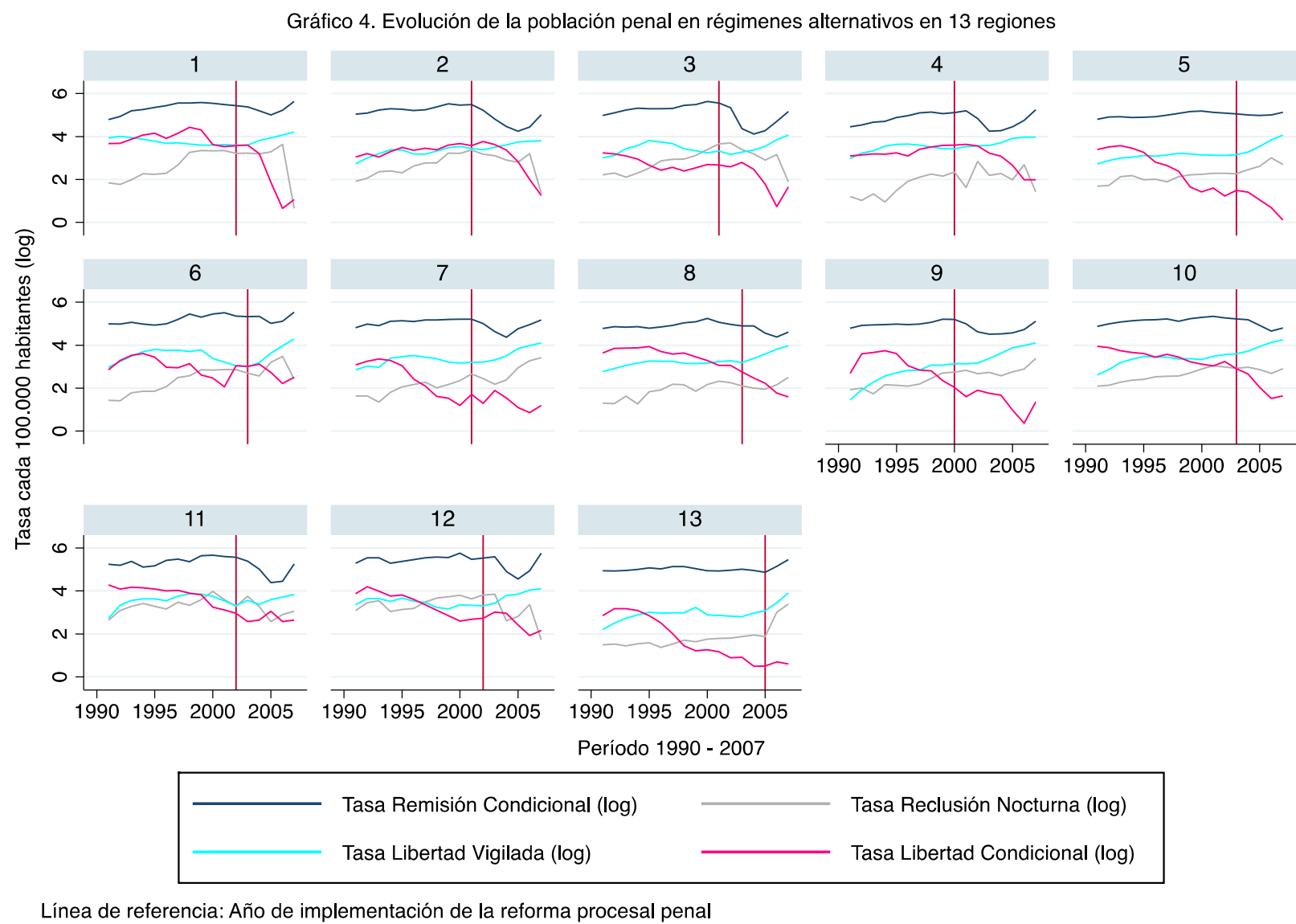

Fuente: Elaboración propia sobre la base de los Compendios Penitenciarios de Gendarmería de Chile e Instituto Nacional de Estadísticas

El gráfico 4 ilustra las tendencias en el uso de las penas sustitutivas de remisión condicional, reclusión nocturna, libertad vigilada y beneficio de libertad condicional. Como se aprecia, la mayoría de las regiones exhibe una tendencia sostenida a la baja en el uso de la libertad condicional hacia el final del periodo de observación. El patrón general permite sugerir que históricamente el uso de la libertad condicional ha disminuido, con independencia de la

\footnotetext{
${ }^{34}$ DAMMERT (2005), p. 40, argumenta que ello se puede deber a su calidad de región limítrofe, con lo que "la alta presencia de tráfico y microtráfico de drogas en esta zona puede ser una explicación a esta situación”.

35 DAMMERT (2005), p. 39.
} 


\section{Polít. Crim. Vol. 16, № 31 (Junio 2021), Art. 3, pp. 62-82 [http://politcrim.com/wp-content/uploads/2021/04/Vol16N31A3.pdf]}

implementación de la reforma procesal penal ${ }^{36}$. Por otra parte, la pena de libertad vigilada muestra una leve tendencia hacia el alza en todas las regiones, sobre todo al final del período de observación. Las tasas de reclusión nocturna disminuyen notoriamente en las regiones 1 , $2,3,4,6,11$ y 12 , pero aumenta en las regiones 7, 8, 9 y 13. Por lo tanto, es incierto si la reforma procesal penal tiene algún efecto en este régimen. Finalmente, los gráficos revelan que la pena de remisión condicional constituye la medida de mayor utilización en todas las regiones y de manera sostenida en el tiempo, aunque algunas regiones presentan discontinuidad en su uso. Como se puede ver, luego de la reforma la curva de remisión condicional baja abruptamente para luego subir. Este patrón es claramente visible en las regiones $1,2,3,4,7,9,11$ y 12 . Es posible sugerir que la reforma probablemente haya tenido un efecto significativo en la medida de remisión condicional.

\subsection{Modelos estadísticos}

La información visual provista por los gráficos presentados en la sección anterior entrega indicios sobre la plausibilidad de las hipótesis bajo análisis. ${ }^{37}$ Sin embargo, la única manera de testear estadísticamente los efectos de la reforma exige utilizar un modelo matemático que compare los efectos de (a) el paso del tiempo y (b) la implementación de la reforma procesal penal en la población penal y considere que (c) cada una de las regiones bajo análisis tiene un comportamiento y año particular de implementación de la reforma.

En nuestro estudio evaluamos estadísticamente los efectos de la reforma procesal penal usando datos de panel (donde las regiones entregan información consecutiva en varios puntos en el tiempo) y un modelo de regresión linear con el propósito de identificar el efecto de la implementación de la reforma procesal penal y distinguirlo del efecto del paso del tiempo por región. Nuestro modelo asume que las regiones tienen numerosas características particulares que no observamos directamente (efectos fijos). Por lo tanto, comparamos el efecto de la reforma entre regiones antes y después de su implementación y mantenemos la variable regional (por ejemplo, "Región Metropolitana") como si tuviera una influencia constante en el tiempo. La variable 'Tiempo' es analizada como variable continua (donde cada año que pasa cuenta como año adicional ' 1 ') y la variable 'Efecto de la reforma' como variable binaria, donde 0 corresponde al año de implementación y anteriores y 1 corresponde al año siguiente y posteriores a la implementación de la reforma procesal penal. Existen 221 puntos de observación correspondientes a 17 registros (años 1991 a 2007) en 13 regiones.

\footnotetext{
${ }^{36}$ MORALES (2012) p. 129 establece que, en esta época, se da un proceso de aprobación de normas tendientes a endurecer la respuesta punitiva frente a determinados tipos penales, que incluye normas que restringen la aplicación del Decreto Ley 321 que establece el régimen de libertad condicional (por ejemplo, la Ley $\mathrm{N}^{\circ} 19.617$ sobre delitos sexuales). Sobre la disminución, véase también: CUNEO (2018), pp. 216 y 217.

${ }^{37}$ Las hipótesis de investigación (H1) y las hipótesis nulas (H0) de estas tres posturas se resumen a continuación. Tesis histórica H1: La población penal total en Chile ha aumentado desde los años ochenta en adelante con independencia de la implementación de la reforma procesal penal y diferencias regionales. H0: No existe efecto del paso del tiempo en las tasas de población penal.

Tesis del incremento H1: La población penal en Chile ha experimentado un drástico aumento a consecuencia de la implementación de la reforma procesal penal. H0: La reforma no influyó en las tasas de población penal. Tesis garantista H1: La reforma procesal penal contribuyó a disminuir las tasas de población penal procesada y detenida. H0: La reforma no influyó en las tasas de población penal procesada y detenida.
} 
La reforma en la mayoría de las regiones fue implementada entre los meses de octubre y diciembre, por esta razón contabilizamos la implementación de la reforma con un lag de un año. Es decir, en nuestro modelo identificamos los efectos inmediatos de la reforma a un año de su implementación. Los resultados que entregamos son los efectos a nivel nacional, pero considerando el carácter regional de los datos. En síntesis, el coeficiente 'Tiempo' captura el efecto de cada año adicional desde 1991 hasta 2007. El coeficiente 'Efecto de la Reforma' captura el efecto de la reforma a un año de su implementación. ${ }^{38}$

Tabla 3. Efectos de la reforma procesal penal en tasas de población penal según régimen.

\begin{tabular}{|c|c|c|c|c|c|c|}
\hline & $\begin{array}{c}(1) \\
\text { Tasa } \\
\text { Cautelados }\end{array}$ & $\begin{array}{c}\text { (2) } \\
\text { Tasa } \\
\text { Condenados }\end{array}$ & $\begin{array}{c}\text { (3) } \\
\text { Tasa } \\
\text { Remisión } \\
\text { Condicional } \\
\end{array}$ & $\begin{array}{c}\text { (4) } \\
\text { Tasa } \\
\text { Reclusión } \\
\text { Nocturna } \\
\end{array}$ & $\begin{array}{c}(5) \\
\text { Tasa Libertad } \\
\text { Vigilada }\end{array}$ & $\begin{array}{c}(6) \\
\text { Tasa } \\
\text { Libertad } \\
\text { Condicional } \\
\end{array}$ \\
\hline Tiempo & $\begin{array}{c}1.018 * * \\
(0.459)\end{array}$ & $\begin{array}{c}9.282 * * * \\
(0.969)\end{array}$ & $\begin{array}{c}5.764 * * * \\
(0.781)\end{array}$ & $\begin{array}{c}1.042 * * * \\
(0.149)\end{array}$ & $\begin{array}{c}0.870 * * * \\
(0.187)\end{array}$ & $\begin{array}{c}-2.148 * * * \\
(0.216)\end{array}$ \\
\hline $\begin{array}{l}\text { Efecto de la } \\
\text { reforma }\end{array}$ & $\begin{array}{c}-43.14 * * * \\
(4.978)\end{array}$ & $\begin{array}{c}11.91 \\
(10.50)\end{array}$ & $\begin{array}{c}-86.12 * * * \\
(8.453)\end{array}$ & $\begin{array}{c}-4.721 * * * \\
(1.614)\end{array}$ & $\begin{array}{c}7.012 * * * \\
(2.026)\end{array}$ & $\begin{array}{c}0.972 \\
(2.338)\end{array}$ \\
\hline Constante & $\begin{array}{c}99.21 * * * \\
(19.94)\end{array}$ & $\begin{array}{l}55.39 * * \\
(26.01)\end{array}$ & $\begin{array}{c}143.2 * * * \\
(10.47)\end{array}$ & $\begin{array}{c}7.236 * * * \\
(2.346)\end{array}$ & $\begin{array}{c}22.49 * * * \\
(2.234)\end{array}$ & $\begin{array}{c}42.17 * * * \\
(3.316)\end{array}$ \\
\hline Observaciones & 221 & 221 & 221 & 221 & 221 & 221 \\
\hline $\begin{array}{l}\text { Número de } \\
\text { regiones }\end{array}$ & 13 & 13 & 13 & 13 & 13 & 13 \\
\hline
\end{tabular}

$$
\begin{gathered}
\text { Errores estándar en paréntesis } \\
* * * \mathrm{p}<0.01, * * \mathrm{p}<0.05, * \mathrm{p}<0.1 \\
\text { Cautelados: Incluye población procesada y detenida }
\end{gathered}
$$

Fuente: Elaboración propia sobre la base de los Compendios Penitenciarios de Gendarmería de Chile e Instituto Nacional de Estadísticas

Los números indican que por cada año que ha transcurrido en el período 1991 hasta la implementación de la reforma (variable 'Tiempo'), se asocia un incremento significativo (indicado con *) en varios regímenes procesales. Desde 1991 ha aumentado significativamente la población cautelada (1.02 cada año), condenada (9.28), sometida a remisión condicional (5.76), en reclusión nocturna (1.04) y libertad vigilada (0.87) por cada cien mil habitantes. Por el contrario, el uso de la medida de libertad condicional ha disminuido sostenidamente en el tiempo (-2.15). Las tendencias muestran que existe un

\footnotetext{
${ }^{38}$ Entonces, por ejemplo, si en la cuarta región la reforma fue implementada el año 2000, nuestro modelo asume que la reforma tiene sus primeros efectos visibles al año 2001. Si la diferencia entre el período 'cero' (año 2000) y el período ' 1 ' (año 2001) es significativa, entonces la reforma tuvo un efecto directo en la población penal. En todo caso, el análisis de sensibilidad a dos años de la reforma entrega resultados que no modifican nuestras conclusiones.
} 
incremento sostenido y consistente al alza de la población penal que se origina desde 1991 . Esta tendencia puede tener origen con anterioridad.

Nótese que por cada año que ha transcurrido en el período 1991-2007, se asocia un aumento en las tasas de población penal cautelada y condenada. Por lo tanto, nuestros modelos estadísticos confirman que el incremento en la población penal es un proceso que tiene lugar a inicios del período de transición a la democracia; en sintonía con los planteamientos de la aquí denominada tesis histórica.

En cuanto a los efectos de la reforma, esta efectivamente disminuyó la tasa de cautelados sometidos a detención y procesamiento en -43.14 por cada 100.000 habitantes. Este resultado respalda los postulados de la denominada tesis garantista, la cual sostiene que la reforma entregó mayores y mejores garantías procesales a la población detenida y sometida a prisión preventiva.

Por otra parte, la reforma disminuyó significativamente el uso de las medidas de remisión condicional (-86.12) y reclusión nocturna (-4.72). Solo la pena de libertad vigilada aumentó luego de la reforma (7.01), circunstancia que coincide con nuestros hallazgos visuales previos.

Los números también aportan evidencia para el análisis de otras aristas del impacto de la reforma procesal penal. De acuerdo con nuestros modelos, en el año inmediatamente posterior a su implementación, la reforma afectó significativamente el uso de las penas sustitutivas. Luego de la reforma disminuyó el uso de las medidas de remisión condicional y reclusión nocturna. Sólo el régimen de libertad vigilada experimenta un aumento por cada año adicional luego de la implementación (3.49).

\section{Discusión y conclusiones}

La reforma procesal penal, implementada en Chile a partir del año 2000, constituye la reforma de mayor envergadura en la historia del sistema de justicia criminal chileno. La magnitud y dirección de sus efectos sobre la población penal son materia de discusión. La denominada tesis 'histórica' sugiere que la población penal en Chile ha aumentado progresivamente desde los años ochenta y que la reforma ha contribuido a este proceso. Por su parte, la facción más crítica, basada en una tesis 'punitivista', considera que la reforma procesal penal dio lugar a la expansión masiva de la población penal e incrementó las tendencias punitivas que antecedían a su implementación. Finalmente, los defensores de la reforma indican que esta habría provocado una disminución de la población penal sometida a medidas cautelares privativas de libertad y postulan una tesis 'garantista'. Hasta ahora, existe escasa evidencia que permita demostrar estadísticamente la plausibilidad de estas tres posturas.

El presente estudio sistematiza la información disponible en los Compendios Penitenciarios de Gendarmería de Chile y provee una aproximación estadística a los datos disponibles sobre población penal. Para ello, analiza las tendencias a nivel regional considerando la población penal detenida, procesada, imputada, condenada, y sujeta a penas sustitutivas y beneficio 
penitenciario de libertad condicional desde los años 1991 a 2007. También evalúa el impacto de la reforma procesal penal a través de un modelo de regresión lineal. La variación de las tendencias en los regímenes procesales a través del tiempo sugiere que la población penal en Chile ha aumentado de manera constante desde la década de 1980. El mayor impacto de la reforma procesal penal se refiere a la disminución de la población cautelada (detenida, procesada e imputada) y la alteración de tendencias respecto a regímenes de penas sustitutivas y beneficios, existiendo una notable tendencia a la baja en el uso de la remisión condicional y la reclusión nocturna.

Hasta nuestro conocimiento, replicar este modelo y mantener continuidad con el período anterior requiere utilizar a los establecimientos penales como unidades de análisis y considerar las modificaciones político-administrativas en el territorio nacional. Como señalábamos, la principal limitación de nuestro estudio consiste en la restricción del período de observación a los años 1991 a 2007. Próximos estudios deberían considerar el efecto de la creación en el año 2007 de dos nuevas regiones y estudiar el efecto de aprendizaje institucional de la reforma procesal penal..$^{39}$ Ellos podrían explicar, por ejemplo, el aumento de la medida de remisión condicional durante el período de consolidación de la reforma. Recomendamos también extender el período bajo estudio y añadir variables explicativas a los modelos estadísticos, tales como género ${ }^{40}$, crecimiento económico regional, expansión de la capacidad estatal e infraestructura pública y estadísticas de criminalidad. ${ }^{41}$

Es posible sostener que la heterogeneidad del uso de penas, medidas alternativas y beneficios penitenciarios revela la ausencia de una política criminal seria y consistente en la legislación chilena. La evolución de las tasas, al ser tan disímil, confirma la aseveración de Silvio Cuneo, quien sostiene que el aumento de la población penal en Chile "no fue el resultado de una política propuesta, investigada, costeada, debatida, y democráticamente acordada, sino que es consecuencia de la convergencia de diversas políticas y decisiones" ${ }^{42}$ El análisis específico de cada una de dichas políticas y sus efectos excede los objetivos del presente trabajo. Por esta razón, futuras investigaciones debieran abordar el estudio de modificaciones legales que puedan haber favorecido el alza de la población penal, ya desde la década del ochenta y anteriores. Entre ellas, el Decreto 597 de 1984 (Reglamento de extranjería), las leyes $\mathrm{N}^{\mathrm{o}} 19.413$ de $1995,19.366$ de $1995,19.449$ de $1996,19.617^{43}$ de 1999 , entre otras, deben ser objeto de minucioso estudio para apreciar su impacto en la población penal.

Nuestro estudio encuentra evidencia para sostener que la reforma procesal penal contribuyó decisivamente a la disminución de la población sometida a medidas cautelares privativas de libertad (detenida, procesada e imputada). No existe evidencia que avale la tesis del incremento, esto es, que la reforma procesal penal conllevó un aumento (inmediato) de la

\footnotetext{
${ }^{39}$ Ver, por ejemplo, WILENMANN (2019), passim.

${ }^{40}$ LARROULET et al. (2020), p. 113.

${ }^{41}$ MATUS Y PEÑA Y LILLO (2012), p. 336.

${ }^{42}$ CUNEO (2018), p. 157.

${ }^{43}$ Estas han sido también mencionadas como influyentes en otros estudios, pero su peso específico en las tendencias de encarcelamiento debe ser aún estudiado más en profundidad. Véase: MORALES (2012), p. 103; CUNEO (2018), pp. 213, 215 y ss.
} 
población condenada. Sin embargo, análisis con datos posteriores al 2007 permiten distinguir el impacto de la reforma inmediatamente después de su implementación, los efectos del aprendizaje institucional a lo largo del tiempo (entendido como el proceso de adaptación en la historia, cultura y prácticas de los operadores del sistema ante un cambio institucional o implementación de una nueva política pública) y las posteriores modificaciones legislativas del proceso penal. ${ }^{44}$ Leyes posteriores, típicamente las denominadas 'leyes de agenda corta', afectan enormemente el tratamiento de la población penal. De esta manera, bien puede ser que a través del tiempo la reforma haya provisto oportunidades para facilitar el crecimiento de la población penal. ${ }^{45}$ Futuras investigaciones, por lo tanto, debieran considerar ampliar el periodo de observación.

\footnotetext{
${ }^{44}$ Tal como sugieren WILENMANN (2019), passim y MORALES (2012), passim.
}

${ }^{45}$ CUNEO (2018), p.203. 


\section{Referencias bibliográficas}

AREVALO, Alejandro (2018): "El impacto de la reforma procesal penal en el sistema penitenciario", en: Revista $93\left(\mathrm{~N}^{\circ} 18\right)$, pp. 48-51.

BAYTELMAN, Andrés; DUCE, Mauricio (2003): "Evaluación de la reforma procesal penal: estado de una reforma en marcha", en: Centro de Estudios de Justicia de las Américas (Santiago, Universidad Diego Portales).

BECKETT, Katherine; WESTERN, Bruce (2001): "Governing Social Marginality. Welfare, Incarceration, and the Transformation of State Policy", en: GARLAND, David (ed.): Mass Imprisonment: Social causes and Consequences (Londres: Sage), pp. 35-47.

CENTRO DE POLÍTICAS PÚBLICAS UC (2017): "Sistema carcelario en Chile: propuestas para avanzar hacia una mayor efectividad y reinserción", en: Temas de la agenda pública (Año 12, $\mathrm{N}^{\circ}$ 93).

CUNEO, Silvio (2008): "Encarcelamiento en Chile. Necesidad de una nueva regulación a nivel constitucional", en: Revista Nova Criminis ( $\left.\mathrm{N}^{\circ} 15\right)$, pp. 1-10.

CUNEO, Silvio (2018): “Cárceles y pobreza, distorsiones del populismo penal” (Santiago: Uqbar).

DAMMERT, Lucía (2005): "Violencia criminal y seguridad ciudadana en Chile", en: CEPAL - SERIE Políticas Sociales ( ${ }^{\circ}$ 109), pp. 1-51.

DOWNES, David (2001): “The Macho Penal Economy, Mass Incarceration in the United States - a European Perspective”, en: Garland, David (ed.): Mass Imprisonment: Social causes and Consequences (Londres: Sage), pp. 51-66.

DUCE, Mauricio; PEREZ, Rogelio (2003): "Citizen security and reform of the criminal justice system in Latin America", en: FRUHLING, Hugo, TULCHIN, Joseph y GOLDING, Heather (ed.): "Crime and Violence in Latin America. Citizen Security, Democracy and the State”, (Baltimore y Londres: Woodrow Wilson University Press, The John Hopkins University Press).

DUCE, Mauricio; RIEGO, Cristián (2016): "La prisión preventiva en Chile: el impacto de la reforma procesal penal y de sus cambios posteriores." (Santiago: CEJA)

GARCÍA, Deborah (2019): "El recurso excesivo al Derecho Penal en España. Realidad y alternativas", en: Polit. Crim. (Vol. 14, No 27), pp. 98 - 121.

GARLAND, David (2001): “The Meaning of Mass Imprisonment”, en: GARLAND, David (ed.): Mass Imprisonment: Social causes and Consequences (Londres: Sage), pp. 13.

HERSANT, Jeanne (2017): "Patronage and rationalization: Reform to criminal procedure and the lower courts in Chile", en: Law \& Social Inquiry (Vol. 42, No 2), pp. 423449.

HOLDERNESS, Clifford (2016): "Problems using aggregate data to infer individual behavior: Evidence from law, finance, and ownership concentration", en: Critical Finance Review (Vol $5 \mathrm{~N}^{\mathrm{o}}$ 1), pp. 1-40.

HORVITZ, María Inés; LÓPEZ, Julián (2005): “Derecho Procesal Penal Chileno” (Santiago: Editorial Jurídica.), Tomo I. 
LANGER, Máximo (2007): "Revolution in Latin American criminal procedure: Diffusion of legal ideas from the periphery", en: The American Journal of Comparative Law (Vol. $\left.55 \mathrm{~N}^{\circ} 4\right)$, pp. 617-676.

LARRAURI, Elena (2009): "La Economía Política del Castigo", en: Revista de Ciencia Penal y Criminología (Vol. 11 Nº), pp. 1-22.

LARRAURI, Elena (2006): "Populismo Punitivo", en: Jueces Para la Democracia, pp. 1521.

LARROULET, Pilar; DROPPELMANN, Catalina; DEL VILLAR, Paloma; DAZA, Sebastián; FIGUEROA, Ana; OSORIO, Victoria (2020): "Who is transitioning out of prison? Characterising female offenders and their needs in Chile", en: International Journal for Crime, Justice and Social Democracy (Vol. $9 \mathrm{~N}^{\circ}$ 1), pp. 112-125.

MACAULAY, Fiona (2007): "Knowledge production, framing and criminal justice reform in Latin America", en: Journal of Latin American Studies (Vol. $39 \mathrm{~N}^{\circ}$ 3) pp. 627 651.

MATUS ACUNA, Jean Pierre; PENA Y LILLO TOLOSA, Ma Carolina (2012): "Comentario crítico a la investigación de Sebastián Salinero Echeverría: ¿Por qué aumenta la población penal en Chile? Un estudio criminológico longitudinal”, en: Ius et Praxis (Vol $18 \mathrm{~N}^{\circ}$ 2), pp. 333-340.

MORALES, Ana María (2012): "La Política Criminal Contemporánea: Influencia en Chile del Discurso de la Ley y el Orden”, en: Polit. Crim. (Vol. 7, No 13), pp. $94-146$.

RIVERA, Iñaki (2017): "Descarcelación, Principios para una Política Pública de Reducción de la Cárcel” (Valencia: Tirant lo Blanch).

SALINERO, Sebastián (2012): “¿Por qué aumenta la población penal en Chile? Un estudio criminológico longitudinal”, en: Ius et Praxis (Año 18, $\mathrm{N}^{\circ} 1$ ), pp. 113-150.

SÁNCHEZ, Mauricio, PIÑOL, Diego (2015): "Condiciones de vida en los centros de privación de libertad en Chile", en: Centro de Estudios en Seguridad Ciudadana Universidad de Chile. Disponible en: https://tinyurl.com/yk2ro32u [visitado el $12 / 12 / 2019]$.

TIEDE, Lydia (2012): “Chile's Criminal Law Reform: Enhancing Defendants' Rights and Citizen Security”, en: Latin American Politics and Society (Vol. 54, N 3 ), pp. 6593.

TORRES, Luis (2015): "Política Criminal y Derechos Humanos en Chile: Notas y desafíos actuales", en: Hors-série (mars 2015) Revue québécoise de droite international, pp. $15-28$.

VENEGAS, Veronica; VIAL, Luis (2008): "Boomerang: Seeking to reform pretrial detention practices in Chile." en: Justice Initiatives: Pretrial Detention (Nueva York: Open Society Institute), pp. 44-56.

WILENMANN, Javier (2019): "Neoliberal Politics and State Modernization in Chilean Penal Evolution", en: Punishment and Society" (Vol. 22, N³), pp. 259-280.

ZIMRING, Franklin (2001), "Imprisonment Rates and the New Politics of Criminal Punishment", en: GARLAND, David (ed.): Mass Imprisonment: Social causes and Consequences, (Londres: Sage), pp. 145-149. 